\title{
Dynamical probe of thermodynamical properties in three- dimensional hairy AdS black holes
}

\author{
De-Cheng Zou ${ }^{1}$, Yunqi Liu ${ }^{2}$, Cheng-Yong Zhang ${ }^{3}$ and Bin WAnG $^{4}$ \\ 1 College of Physical Science and Technology, Yangzhou University - Yangzhou, 225009, China \\ 2 School of Physics, Huazhong University of Science and Technology - Wuhan, 430074, China \\ 3 Center of High Energy Physics, Peking University - Beijing, 100871, China \\ 4 Department of Physics and Astronomy, Shanghai Jiao Tong University - Shanghai 200240, China
}

PACS 04.70.-s - Physics of black holes

PACS 04.25.D- - Numerical relativity

PACS 04.50.Kd - Modified theories of gravity

\begin{abstract}
We study separatively the quasinormal modes (QNM) of electromagnetic perturbations around three-dimensional anti-de Sitter(AdS) black holes in Jordan and Einstein frames, which are related by the conformal transformations and a redefinition of a scalar field. We find that, in the Jordan frame, the imaginary parts of QNM frequencies can reflect the thermodynamical stabilities of hairy black holes, including the possible phase transition between the hairy black hole and BTZ black hole, disclosed by examining the corresponding free energies. Similar results are also uncovered in the Einstein frame. The obtained results further support that the QNM can be a dynamic probe of the thermodynamic properties in black holes.
\end{abstract}

Introduction. - In black hole physics there is a famous no hair theorem. In general, the no-hair theorem 'rules out the black holes coupled with a scalar field in - asymptotically flat spacetimes [1]3, because the scalar ' field can diverge on the horizon and make the black hole become unstable [4. The dawn of constructing a black hole with scalar hair broke on the horizon when a negative cosmological constant was taken into account. For example, AdS black holes with scalar hair in the scalar-tensor theories (STT) have been respectively constructed in three dimensional [5] 10] and higher dimensional spacetimes 11 19. Thermodynamically, AdS black holes with scalar hair are very interesting. It was observed that static black hole with minimally coupled scalar field hair can transform to static BTZ black hole in three dimensional AdS spacetimes 20,21. A second order phase transition was disclosed to occur between the four-dimensional AdS black hole with a minimally coupled scalar field hair (MartinezTroncoso-Zanelli(MTZ) black hole) and the topological black hole with hyperbolic horizon (TBH) [11,20]. Furthermore, phase transitions among charged TBH, charged MTZ black holes [14,22] and other exact hairy black hole solutions [17] in the four-dimensional AdS spacetimes were also uncovered. It is of great interest to generalize the discussions and find more examples on the relation between the dynamical physical phenomenon and its corresponding thermodynamic properties. In particular, the discussion for a rotating black hole in the STT is still lack. In this work, we will concentrate our attention on the rotating hairy AdS black holes in the three-dimensional STT, which was recently reconstructed in 23,24 .

On the other hand, there exist two versions of STT: one version is on the Einstein frame and the other is on the Jordan frame, which can be related to the former by a conformal transformation. One may have a nonminimally coupled scalar in the Jordan frame, while one may have a minimally coupled scalar in the Einstein frame. Here we want to ask whether the intriguing thermodynamical relation between the rotating hairy black holes and rotating BTZ black holes in AdS spacetimes can be reflected in dynamical properties so that it can have some observational signatures to be detected in the both frames. This is our main purpose here. Considering that quasinormal modes of dynamical perturbations are characteristic sounds of black holes, we expect that the black hole phase transitions can be imprinted in the dynamical perturbations in their surrounding geometries through frequencies and damping times of the oscillations. In the last few decades there have been extensive research [25]32, where the relations between thermodynamical phase transitions and 
dynamical perturbations have been discussed. Besides, we will further discuss the static hairy black holes within both frames.

The paper is organized as follows: in the next section, we review the thermodynamical properties of hairy black holes, including the possible phase transitions between the hairy black hole and BTZ black holes in the Jordan and Einstein frames. In the third section, we will disclose that this phase transition can be numerically reflected by the QNM frequencies of perturbations in both frames. We complete the paper with conclusions and discussions in the fourth section.

Thermodynamics of three dimensional AdS Black holes with scalar hair. - In this section, we consider the thermodynamics of hairy black holes in the Einstein and Jordan frames. The action in Einstein frame is given by 23 .

$$
I=\frac{1}{2} \int \mathrm{d}^{3} x \sqrt{-g}\left(R-\nabla_{\mu} \phi \nabla^{\mu} \phi-2 V(\phi)\right),
$$

where $\phi(r)$ is the scalar field and the scalar potential $V(\phi)$ takes the form

$$
\begin{array}{r}
V(\phi)=-\frac{1}{l^{2}} \cosh ^{6}\left(\frac{1}{2 \sqrt{2}} \phi\right)+\frac{1}{l^{2}}\left(1+\mu l^{2}\right) \sinh ^{6}\left(\frac{1}{2 \sqrt{2}} \phi\right) \\
-\frac{\alpha^{2}}{64} \sinh ^{10}\left(\frac{1}{2 \sqrt{2}} \phi\right) \cosh ^{6}\left(\frac{1}{2 \sqrt{2}} \phi\right)\left[\tanh ^{6}\left(\frac{1}{2 \sqrt{2}} \phi\right)\right. \\
\left.-5 \tanh ^{4}\left(\frac{1}{2 \sqrt{2}} \phi\right)+10 \tanh ^{2}\left(\frac{1}{2 \sqrt{2}} \phi\right)-9\right]
\end{array}
$$

with the parameters $\mu, \alpha$, and $l$ related to the negative cosmological constant $\Lambda=-\frac{1}{l^{2}}$. Using a particular conformal factor of the form $\check{g}_{\mu \nu}=\Omega^{2} g_{\mu \nu}$ and $\Omega=\cosh ^{2}(\sqrt{8} \phi)$, and defining a new scalar field $\breve{\phi}(r)=\sqrt{8} \tanh (\sqrt{8} \phi)$ and scalar potential $U(\check{\phi})=V(\phi) \Omega^{-3}$, the action above can be transformed into the corresponding action in the Jordan frame 24

$$
\begin{aligned}
\mathcal{I}= & \frac{1}{2} \int d^{3} x \sqrt{-\check{g}}\left(\check{R}-2 \Lambda-\check{g}^{\mu \nu} \nabla_{\mu} \check{\phi} \nabla_{\nu} \check{\phi}\right. \\
& \left.-\frac{1}{8} \check{R} \check{\phi}^{2}-2 U(\check{\phi})\right)
\end{aligned}
$$

with

$$
\begin{aligned}
U(\check{\phi})= & \frac{1}{512}\left(\frac{1}{l^{2}}+\mu\right) \check{\phi}^{6} \\
& +\frac{\alpha^{2}\left(\check{\phi}^{6}-40 \check{\phi}^{4}+640 \check{\phi}^{2}-4608\right) \check{\phi}^{10}}{512\left(\check{\phi}^{2}-8\right)^{5}} .
\end{aligned}
$$

In the Jordan frame. The action (3) above admits the following solution 24

$$
\begin{aligned}
& d s^{2}=-f(r) d t^{2}+f(r)^{-1} d r^{2}+r^{2}(d \varphi+\omega(r) d t)^{2}(5) \\
& f(r)=\mu B^{2}\left(3+\frac{2 B}{r}\right)+\frac{(3 r+2 B)^{2} \alpha^{2} B^{4}}{r^{4}}+\frac{r^{2}}{l^{2}} \\
& \omega(r)=-\frac{\alpha B^{2}(3 r+2 B)}{r^{3}} .
\end{aligned}
$$

Here the parameter $B$ is related to the scalar field

$$
\check{\phi}(r)= \pm \sqrt{\frac{8 B}{r+B}} .
$$

For this rotating hairy black hole, the mass and angular momentum can be calculated by adopting the Brown-York method 33. The quasilocal mass $m(r)$ at $r$ takes the form 3436

$$
m(r)=\sqrt{f(r)} E(r)-j(r) \omega(r),
$$

where $E(r)=2\left(\sqrt{f_{0}(r)}-\sqrt{f(r)}\right)$ is the quasilocal energy at $r$ and $j(r)=\frac{d \omega(r)}{d r} r^{3}$ is the quasilocal angular momentum. Here, $f_{0}(r)=\frac{r^{2}}{l^{2}}$ is a background metric function that determines the zero of the energy. As a result, the mass and angular momentum of the black hole can be obtained as

$$
M \equiv \lim _{r \rightarrow \infty} m(r)=-3 \mu B^{2}, \quad J \equiv \lim _{r \rightarrow \infty} j(r)=6 \alpha B^{2} .
$$

Thus the black hole metric coefficient [Eq. (5)] can be rewritten as

$$
f(r)=-M\left(1+\frac{2 B}{3 r}\right)+\frac{r^{2}}{l^{2}}+\frac{(3 r+2 B)^{2} J^{2}}{36 r^{4}}
$$

The condition $-\mu \geq \frac{2 \alpha}{l}$ is needed to protect the cosmic censorship, which puts the constraint $\frac{M}{J} \geq \frac{1}{l}$ for this black hole.

From $f\left(r_{+}\right)=0$, the horizon radius $r_{+}$can be expressed as $r_{+}=B \times \theta$, where $\theta$ is related to the parameters $\mu, \alpha$ and $l$ 24. Below, we set $l=1$ for simplicity. Then the mass, temperature, heat capacity, angular momentum and entropy of the rotating hairy black hole are obtained as

$$
\begin{aligned}
& M=\frac{J^{2}(2+3 \theta)^{2}+36 r_{+}^{4} \theta^{2}}{12 r_{+}^{2} \theta(2+3 \theta)}, \\
& T=\frac{(1+\theta)\left[36 r_{+}^{4} \theta^{2}-J^{2}(2+3 \theta)^{2}\right]}{24 \pi r_{+}^{3} \theta^{2}(2+3 \theta)}, \\
& C=\frac{32 \pi^{2} r_{+}^{4} \theta(2+3 \theta) T}{(1+\theta)^{2}\left[12 \theta^{2} r_{+}^{4}+J^{2}(2+3 \theta)^{2}\right]} \\
& J=M a, \quad a=-\frac{2 \alpha}{\mu}, \quad S=\frac{4 \pi \theta r_{+}}{1+\theta} .
\end{aligned}
$$

In addition, by introducing a family of locally nonrotating observers, the angular velocity for these observers that move on orbits with constant $r$ is given by 37,38.

$$
\Omega=-\frac{g_{t \psi}}{g_{\psi \psi}}=-\omega(r)=\frac{(3 r+2 B) J}{6 r^{3}} .
$$

When approaching the black hole horizon, the angular velocity $\Omega_{+}$turns out to be

$$
\begin{aligned}
\Omega_{+} & =-\omega\left(r_{+}\right)=\frac{\left(3 r_{+}+2 B\right) J}{6 r_{+}^{3}} \\
& =\frac{(2+3 \theta) J}{6 \theta r_{+}^{2}} .
\end{aligned}
$$


Combining these quantities, $M, T$, and $\Omega_{+}$, we can verify that the first law of thermodynamics holds in this case

$$
d M=T d S+\Omega_{+} d J
$$

and the Smarr relation can be found

$$
M-\Omega_{+} J=\frac{1}{2} T S .
$$

In general, thermodynamical stabilities can be disclosed by examining the free energy. It has the following expression in the canonical ensemble (fixed $J$ )

$$
F_{r}=M-T S=\frac{J^{2}(2+3 \theta)^{2}-12 r_{+}^{4} \theta^{2}}{4 r_{+}^{2} \theta(2+3 \theta)} .
$$

Here the subscript " $r$ " denotes the rotating black hole. Firstly, we consider the static case $(J=0)$. Using the relation (11), the free energy of the static hairy black hole can be written as

$$
F_{s}=-\frac{\theta(2+3 \theta)}{3(1+\theta)^{2}}(2 \pi T)^{2}=-\xi(\theta)(2 \pi T)^{2} .
$$

Here the subscript " $s$ " denotes the static black hole. It is easily verified that $\xi(\theta)$ is a monotone increasing function of $\theta$ because of $\frac{d \xi(\theta)}{d \theta}>0$. Therefore the free energy $F_{s}$ becomes more negative with the increasing of $\theta$, so that this static hairy black hole becomes more stable, see fig. 1(a). For a rotating hairy black hole, we can rewrite eqs. (11) (16) as

$$
T=\frac{F_{r} \sqrt{F_{r}^{2}+3 J^{2}}-F_{r}^{2}-J^{2}}{\sqrt{2 \xi(\theta)}\left(\sqrt{F_{r}^{2}+3 J^{2}}-F_{r}\right)^{3 / 2}} \equiv \frac{\Upsilon\left(F_{r}, J\right)}{\sqrt{\xi(\theta)}} .
$$

Consider the function $\Upsilon\left(F_{r}, J\right)$, we obtain

$$
\begin{gathered}
\frac{\partial \Upsilon\left(F_{r}, J\right)}{\partial F_{r}}=\frac{2 F_{r} \sqrt{F_{r}^{2}+3 J^{2}}-\left(2 F_{r}^{2}+3 J^{2}\right)}{2 \sqrt{2} \pi\left(\sqrt{F_{r}^{2}+3 J^{2}}-F_{r}\right)^{5 / 2}}<0, \\
\frac{\partial \Upsilon\left(F_{r}, J\right)}{\partial J}=\frac{J\left(\sqrt{F_{r}^{2}+3 J^{2}}-F_{r}\right)}{2 \sqrt{2} \pi\left(\sqrt{F_{r}^{2}+3 J^{2}}-F_{r}\right)^{5 / 2}}>0,
\end{gathered}
$$

which imply that $\Upsilon\left(F_{r}, J\right)$ is a monotone increasing function of $J$ or a monotone decreasing function of $F_{r}$. Consequently, the free energy $F_{r}$ with any fixed temperature $T$ will reach smaller values when taking smaller values of $J$ or larger values of $\theta$ because of the monotone increasing function $\xi(\theta)$ of $\theta$, or vice versa. These properties are shown in fig. 1(b)(c). In addition, the function $\xi(\theta)$ increases more quickly than $\Upsilon\left(F_{r}, J\right)$ of $J$ on account of $\frac{d^{2} \Upsilon\left(F_{r}, J\right)}{d J^{2}}<0$ and $\frac{d^{2} \xi(\theta)}{d \theta^{2}}>0$.

Another solution of this theory is the rotating BTZ black hole, which is obtained from the action (3) provided that $\check{\phi}(r)=0$. The corresponding metric is

$$
\begin{gathered}
d s^{2}=-f(\rho) d t^{2}+f(\rho)^{-1} d \rho^{2}+\rho^{2}(d \varphi+\Omega(\rho) d t)^{2}, \\
f(\rho)=-\hat{M}+\rho^{2}+\frac{\hat{J}^{2}}{4 \rho^{2}}, \quad \Omega(\rho)=-\frac{\hat{J}^{2}}{2 \rho^{2}} .
\end{gathered}
$$

The thermodynamic quantities in the canonical ensemble(fixed $J$ ), such as the temperature, mass, heat capacity, entropy, angular velocity and free energy of the rotating BTZ black hole are given by

$$
\begin{aligned}
& \hat{M}=\rho_{+}^{2}+\frac{\hat{J}^{2}}{4 \rho_{+}^{2}} \quad, \hat{\Omega}_{+}=\frac{\hat{J}}{2 \rho_{+}^{2}}, \quad \hat{S}=4 \pi \rho_{+}, \\
& \hat{T}=\frac{4 \rho_{+}^{4}-\hat{J}^{2}}{8 \pi \rho_{+}^{3}}, \quad \hat{C}=\frac{32 \pi^{2} \rho_{+}^{4} T}{4 \rho_{+}^{4}+3 \hat{J}^{2}}, \\
& \hat{F}_{r}=\frac{3 \hat{J}^{2}-4 \rho_{+}^{4}}{4 \rho_{+}^{2}} .
\end{aligned}
$$

We can also rewrite eq. (24) into

$$
\hat{T}=\frac{\hat{F}_{r} \sqrt{\hat{F}_{r}^{2}+3 \hat{J}^{2}}-\hat{F}_{r}^{2}-\hat{J}^{2}}{\sqrt{2}\left(\sqrt{\hat{F}_{r}^{2}+3 \hat{J}^{2}}-\hat{F}_{r}\right)^{3 / 2}}=\hat{\Upsilon}\left(\hat{F}_{r}, \hat{J}\right) .
$$

Clearly, the function $\hat{\Upsilon}\left(\hat{F}_{r}, \hat{J}\right)$ shares the same form as the function $\Upsilon\left(F_{r}, J\right)$, whose function characteristic has been discussed above.

Notice that the specific heat (eq. (11)) of the hairy black hole is always positive, which means that the scalar black hole can always reach thermal equilibrium with a heat bath. Moreover, a BTZ black hole with a vanishing scalar field can also be at equilibrium with the heat bath. This raises the question of whether the hairy black hole could decay into the BTZ black hole. In order to find possible phase transition between the hairy and undressed states, we introduce the matching for the temperature $T=\hat{T}$ and angular momentum $J=\hat{J}$, and obtain

$$
\hat{\Upsilon}\left(\hat{F}_{r}, J\right)=\frac{\Upsilon\left(F_{r}, J\right)}{\sqrt{\xi(\theta)}} .
$$

Taking into account $0<\sqrt{\xi(\theta)}<1$, we can obtain

$$
\hat{\Upsilon}\left(\hat{F}_{r}, J\right)>\Upsilon\left(F_{r}, J\right) \Rightarrow \hat{F}_{r}<F_{r} .
$$

This indicates that, with same temperature $T$ and angular momentum $J$, the rotating BTZ black hole always has smaller free energy which is a thermodynamically more preferred phase compared to the rotating hairy black hole. More importantly, this conclusion is universal and irrelevant to any specific positive values of $J$ and $\theta$. Thus, there exists a possible phase transition for this rotating hairy black hole to become rotating BTZ black hole, provided that there are some thermal fluctuations. In static case, we can directly calculate the difference of free energies from eqs. (17) (24)

$$
\Delta F_{s}=F_{s}-\hat{F}_{s}=\frac{3+4 \theta}{3(1+\theta)^{2}}(2 \pi T)^{2} .
$$

Since the constant $\theta$ always remains positive 24, we have $\Delta F_{s}>0$, namely $F_{s}>\hat{F}_{s}$. So, the static BTZ black hole is more thermodynamically preferred. When $\theta \rightarrow+\infty$, $\Delta F_{s}$ approaches zero, then the free energies of two black holes become consistent. 

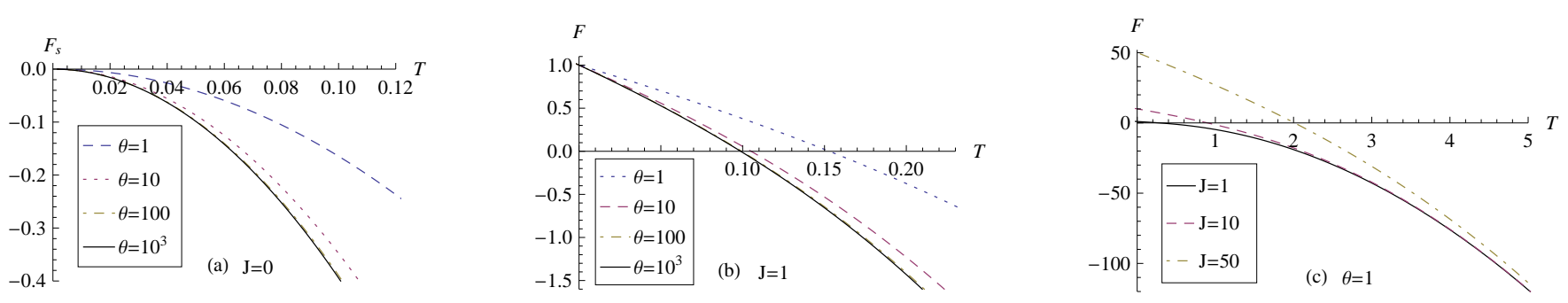

Fig. 1: The free energies $F$ of hairy black hole versus the temperature $T$ in the Jordan frame.

In the Einstein frame. Now we generalize the above discussions to the three-dimensional hairy black holes in the Einstein frame. As shown in [23, the action (11) admits the following solution

$$
\begin{gathered}
\mathrm{d} s^{2}=-f_{1}(H) \mathrm{d} t^{2}+\frac{\mathrm{d} r^{2}}{f_{2}(H)}+r^{2}(\mathrm{~d} \psi+\omega(H) \mathrm{d} t)^{2} \\
f_{1}(H)=\frac{H^{2} f(H)}{(H+B)^{2}}, \quad f_{2}(H)=\frac{(H+2 B)^{2} f(H)}{(H+B)^{2}}
\end{gathered}
$$

where the function $f(H)$ and $\omega(H)$ read as

$$
\begin{gathered}
f(H)=3 \mu B^{2}+\frac{2 \mu B^{3}}{H}+\frac{\alpha^{2} B^{4}(3 H+2 B)^{2}}{H^{4}}+\frac{H^{2}}{l^{2}}, \\
\omega(H)=-\frac{\alpha B^{2}(3 H+2 B)}{H^{3}} .
\end{gathered}
$$

Here the parameter $B$ is related to the scalar field

$$
\begin{aligned}
& \phi(r)=2 \sqrt{2} \operatorname{arctanh} \sqrt{\frac{B}{H(r)+B}}, \\
& H(r)=\frac{1}{2}\left(r+\sqrt{r^{2}+4 B r}\right) .
\end{aligned}
$$

From $f(H)=0$, the horizon radius $H_{+}$can be expressed as $H_{+}=B \times h$, where $h$ is related to the parameters $\mu, \alpha$ and $l[23$. Moreover, the corresponding thermodynamical quantities of the rotating hairy black hole are obtained as

$$
\begin{aligned}
M & =\frac{J^{2}(3 h+2)^{2}+36 h^{2} H_{+}^{4}}{12 h(3 h+2) H_{+}^{2}}, \quad \Omega_{+}=\frac{(3 h+2) J}{6 h H_{+}^{2}}, \\
T & =\frac{(1+h)\left[36 H_{+}^{4} h^{2}-J^{2}(2+3 h)^{2}\right]}{24 \pi h^{2}(2+3 h) H_{+}^{3}}, \quad S=\frac{4 \pi h H_{+}}{(h+1)}, \\
C & =\frac{32 \pi^{2} H_{+}^{4} h(2+3 h) T}{(1+h)^{2}\left[12 h^{2} H_{+}^{4}+J^{2}(2+3 h)^{2}\right]}, \\
F_{r} & =\frac{J^{2}(3 h+2)^{2}-12 h^{2} H_{+}^{4}}{4 h(3 h+2) H_{+}^{2}} .
\end{aligned}
$$

Notice that these expressions are similar to their counterparts (11) (13) (16) for the hairy black hole in the Jordan frame disclosed in fig. 11 This implies that the static/rotating hairy black hole in the Einstein frame possesses the same thermodynamical properties, compared with the static/rotating hairy black holes in the Jordan frame. Moreover, there also exists a nonvanishing possibility for a static/rotating hairy black hole to decay into a static/rotating BTZ black hole. In other words, we find that the black hole thermodynamical stabilities in both frames are equivalent.

\section{QNMs of electromagnetic field perturbations. -}

In the Jordan frame. To reflect the thermodynamical stabilities in dynamical perturbations, we calculate the QNMs of electromagnetic field perturbations around this hairy black hole. The electromagnetic perturbations are governed by Maxwell's equations

$$
F_{; \nu}^{\mu \nu}=0, \quad F_{\mu \nu}=\partial_{\mu} A_{\nu}-\partial_{\nu} A_{\mu}
$$

As the background is circularly symmetric, it would be advisable to expand $A_{\mu}$ in 3 -dimensional vector spherical harmonics

$$
A_{\mu}(t, r, \varphi)=\left(\begin{array}{c}
P(r) \\
S(r) \\
Q(r)
\end{array}\right) e^{-i \omega t+i m \varphi}
$$

where $m$ is our angular quantum number and $\omega$ is the frequency. With the ansatz (5), we substitute the expansion (34) into Maxwell's equations (33), and obtain

$$
\begin{aligned}
F_{; \nu}^{\varphi \nu}= & \frac{d}{d r}\left(r N(r) \omega(r)^{2}+r K(r) \omega(r)\right) \\
& -\frac{d}{d r}\left(\frac{f(r) N(r)}{r}\right)+\frac{\omega r(m P(r)+\omega Q(r))}{r^{2} f(r)}=0, \\
F_{; \nu}^{r \nu}= & -m r^{2} N(r) \omega(r)+\left(m f(r)-\omega r^{2} \omega(r)\right) N(r) \\
& -(\omega+m \omega(r)) r^{2} K(r)=0, \\
F_{; \nu}^{t \nu}= & \frac{d}{d r}(r N(r) \omega(r)+r K(r)) \\
& -\frac{m(m P(r)+\omega Q(r))}{r f(r)}=0,
\end{aligned}
$$

where $N(r)=i m S(r)-\frac{d Q(r)}{d r}$ and $K(r)=i \omega S(r)+\frac{d P(r)}{d r}$.

Based on these equations, a second order differential radial equation for the electromagnetic perturbation can 
be derived as 1

$$
\begin{aligned}
\psi^{\prime \prime}(r)+ & \frac{f^{\prime}(r) \psi^{\prime}(r)}{f(r)}+\left[\frac{(m \omega(r)+\omega)^{2}}{f^{2}(r)}+\frac{1}{4 r^{2}}\right. \\
& \left.-\frac{m^{2}}{r^{2} f(r)}-\frac{f^{\prime}(r)}{2 r f(r)}\right] \psi(r)=0,
\end{aligned}
$$

where the wavefunction $\psi(r)$ is set to be a combination of functions $f(r), \omega(r)$ and $N(r)$

$$
\psi(r)=\frac{f(r)}{r^{1 / 2}} \frac{\omega N(r)}{m \omega(r)+\omega} .
$$

Defining the tortoise coordinate $d r_{*}=d r / f(r)$, the radial wave equation can be expressed as

$$
\partial_{r_{*}}^{2} \psi(r)+V(r) \psi(r)=0
$$

where the generalized potential $V(r)$ is given by

$V(r)=(m \omega(r)+\omega)^{2}+\frac{f(r)^{2}}{4 r^{2}}-\frac{m^{2} f(r)}{r^{2}}-\frac{f^{\prime}(r) f(r)}{2 r}$.

At the AdS boundary $r \rightarrow+\infty$, the generalized potential $V(r)$ diverges. Then, we need $\psi(r)=0$ [39]. From eq. (36), the incoming wavefunction $\psi(r)$ near the horizon reads as

$$
\psi(r) \sim\left(r-r_{+}\right)^{-\frac{i \kappa}{4 \pi T}}, \quad \kappa=\omega+m \omega\left(r_{+}\right) .
$$

Defining $\quad \psi(r) \quad$ as $\quad \varphi(r) \exp \left[-i \int \frac{\kappa}{f(r)} d r\right]$, where $\exp \left[-i \int \frac{\kappa}{f(r)} d r\right]$ asymptotically approaches to the ingoing wave near horizon, eq. (36) becomes

$$
\begin{aligned}
& 4 r^{2} f(r) \varphi^{\prime \prime}(r)+4 r^{2} \varphi^{\prime}(r)\left[f^{\prime}(r)-2 i \kappa\right]+\left[f(r)-2\left(2 m^{2}\right.\right. \\
& \left.\left.+r f^{\prime}(r)\right)+\frac{4 r^{2}\left((m \omega(r)+\omega)^{2}-\kappa^{2}\right)}{f(r)}\right] \varphi(r)=0
\end{aligned}
$$

so that $\varphi\left(r_{+}\right)=1$ in case of $r \rightarrow r_{+}$. Then we can numerically solve eq. (40) and find the QNM frequencies under the boundary conditions by adopting the shooting method. The lowest QNM frequencies of perturbations with $m=1$ around this static and rotating hairy black holes at $T=0.5$ are shown in fig. 2. Fixing the angular momentum, the imaginary parts of both QNM frequencies become more negative with the increase of $\theta$, and then almost remains unchanged when $\theta$ is big enough. Moreover, the rotating hairy black hole with smaller values of $J$ is

\footnotetext{
${ }^{1}$ For a massless scalar field perturbation around the three dimensional hairy black hole in the Jordan frame, the Klein-Gordon equation is given by

$$
\frac{1}{\sqrt{-g}} \partial_{\mu}\left(g^{\mu \nu} \sqrt{-g} \partial_{\nu}\right) \Psi=0
$$

Introducing the following ansatz for the field

$$
\Psi=\frac{\psi(r)}{r^{1 / 2}} e^{-i \omega t+i m \varphi}
$$

the radial equation for scalar field perturbation takes the same form as eq. (36).
}

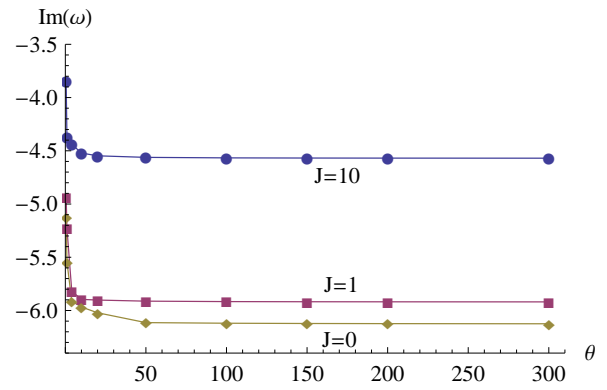

Fig. 2: Lowest QNM frequencies of electromagnetic perturbations with $m=1$ around hairy black holes at $T=0.5$ in the Jordan frame.

more stable, since the imaginary parts of QNM frequencies are more negative. These results exactly reflect the thermodynamic stabilities of the static and rotating hairy black holes disclosed in fig. 1.

On the other hand, the QNM frequencies for BTZ and hairy black holes with $m=1$ are shown in fig. 3. One can see that the numerical results of QNM frequencies agree excellently with analytical ones [40] in (rotating and static) BTZ black holes. Moreover, with the growth of $\theta$, the imaginary parts for a hairy black hole become more negative, and then approach the curves of a BTZ back hole. These results match the thermodynamical stability analysis above.

In the Einstein frame. Now we calculate the QNMs of electromagnetic field perturbations around this rotating/static hairy black hole in the Einstein frame. The differential equation for electromagnetic perturbation in the Einstein frame can be obtained as 2

$$
\begin{aligned}
& \varphi^{\prime \prime}(H)+\varphi^{\prime}(H)\left[\frac{f^{\prime}(H)}{f(H)}-2 i \kappa \zeta\right]+\left[\frac{4 B+H}{4 H(B+H)^{2}}\right. \\
& -\frac{m^{2}}{f(H)}-\frac{(B+H) f^{\prime}(H)}{2 H^{2} \zeta f(H)}+\frac{(m \omega(H)+\omega)^{2}}{f(H)^{2}} \\
& \left.-\frac{\kappa^{2} \zeta^{2}}{f(H)^{2}}+\frac{2 i B^{2} \zeta^{2} \kappa}{(B+H)^{3} f(H)}\right] \varphi(H)=0
\end{aligned}
$$

with

$$
\zeta=\frac{(B+H)^{2}}{H(2 B+H)}, \quad \kappa=\frac{(2+h) h}{(1+h)^{2}}\left(\omega+\omega\left(H_{+}\right) .\right.
$$

Here function $\varphi(H)$ equals to 1 in case of $H \rightarrow H_{+}$, and vanishes at AdS infinity. By calculating numerically eq. (41) under the boundary conditions, we find that, in the Einstein frame, the behaviors of lowest QNM frequencies are consistent with the thermodynamic stabilities of these hairy black holes disclosed in figs. 415.

Conclusions and Discussions. - By calculating the QNMs of electromagnetic perturbations, we found that the

\footnotetext{
${ }^{2}$ For a massless scalar field perturbation around the three dimensional hairy black hole in the Einstein frame, it will be found that the perturbation equation takes the same form as eq. (41).
} 

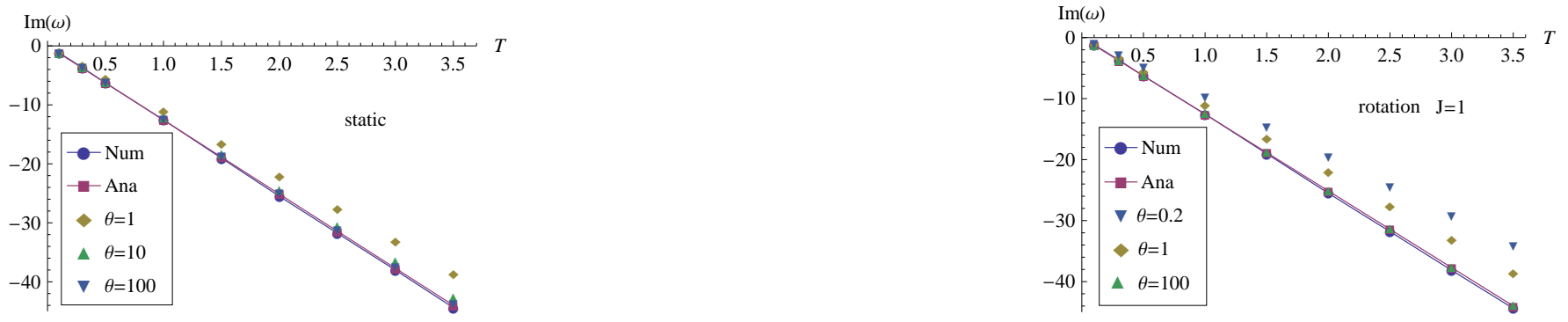

Fig. 3: Lowest QNM frequencies of electromagnetic perturbations with $m=1$ for hairy black holes at $T=0.5$ in the Jordan frame.
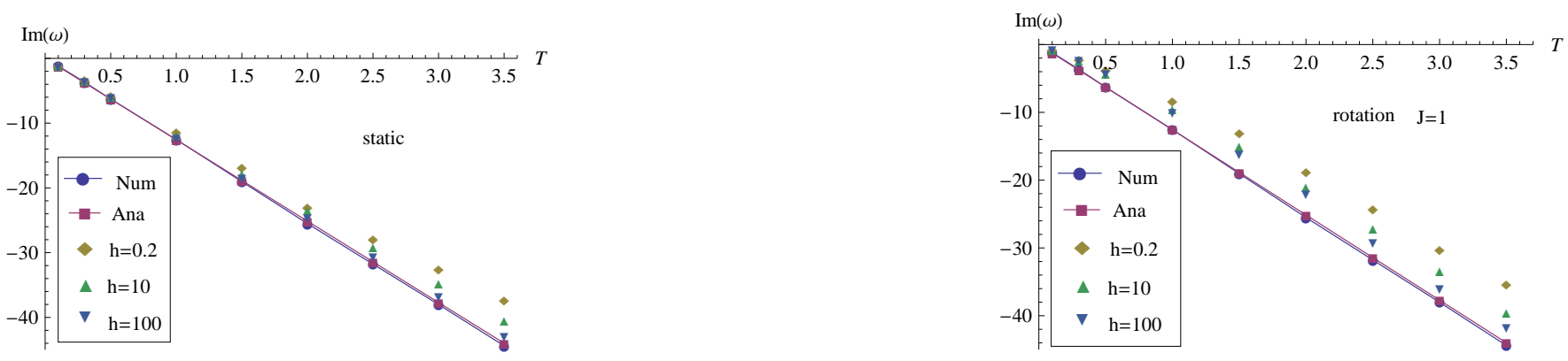

Fig. 5: Lowest QNM frequencies of electromagnetic perturbations with $m=1$ for hairy black holes at $T=0.5$ in the Einstein frame.

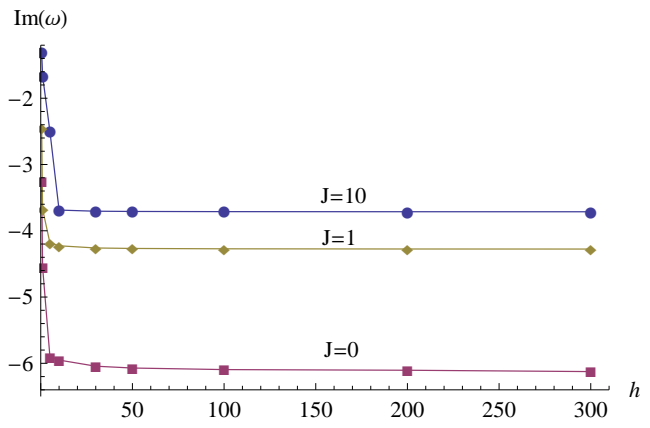

Fig. 4: Lowest QNM frequencies of electromagnetic perturbations with $m=1$ around hairy black holes at $T=0.5$ in the Einstein frame.

rotating hairy black holes with larger values of $\theta$ (in the Jordan frame) or $h$ (in the Einstein frame), and smaller values of $J$ can be more dynamically stable objects in the canonical ensemble. The disclosed dynamical stability properties are consistent with the thermodynamical stability for these hairy black holes. On the other hand, compared with the rotating hairy black hole, the rotating BTZ black hole is dynamically more stable in both frames, due to the fact that it has larger absolute imaginary QNM frequencies. This property is again in agreement with the fact that the rotating BTZ black hole shares lower free energy and is more thermodynamically preferred. Similar observations on the dynamical phenomenon and its relation to thermodynamics have been disclosed between the static hairy black hole and its BTZ black hole counterpart in both frames. Considering that the QNM is expected to be detected and has strong astrophysical interest, we hope that the dynamical probe can really present us the observational signature of the thermodynamic property of black holes.

Recently, Yun Soo Myung et al. 41 found that the black hole (in)stability is independent of the frame which shows that the two frames are equivalent to each other. Similar equivalence of the Einstein and Jordan frames also appeared in the Higgs inflation 42 . However, S.Bahamonde et al. 43 found the frame dependence of $F(R)$ gravity singularities in Jordan and Einstein Frames. Moreover, Faraoni et al. 44, 45 showed that, with some proper interpretation, the two versions are actually equivalent at the classical level, while inequivalent at the quantum level. Here our present work provides a example to support the claim of the equivalence of two frames by means of the hairy black hole thermodynamics and corresponding dynamical properties in both frames. However, whether the two versions of STT are equivalent or not in the classical gravity is still generates plenty of controversy. This deserves research in further.

$* * *$

This work is supported by Natural Science Foundation of Higher Institutions of Jiangsu Province (Grant No.16KJB140020) and Natural Science Foundation for Youths of Jiangsu Province (Grant No.BK20160452). 


\section{REFERENCES}

[1] Bekenstein J. D., Annals Phys., 82 (1974) 535,Annals Phys., 91 (1975) 75.

[2] Xanthopoulos B. C. and Dialynas T. E., J. Math. Phys., 33 (1992) 1463.

[3] Kuimcik C., J. Math. Phys., 34 (1993) 1914.

[4] Bronnikov K. A. and Kireev Y. N., Phys. Lett. A, 67 (1978) 95.

[5] Martinez C. and Zanelli J.,, Phys. Rev. D, 54 (1996) 3830.

[6] Henneaux M., Martinez C., Troncoso R. and Zanelli J., Phys. Rev. D, 65 (2002) 104007.

[7] Xu W. and Zhao L., Phys. Rev. D, 87 (2013) 124008.

[8] Zhao L., Xu W. and Zhu B., Commun. Theor. Phys., 61 (2014) 475 .

[9] Mazharimousavi S. H. and Halilsoy M., Mod. Phys. Lett. A, 30 (2015) 1550177.

[10] Banados M. and Theisen S., Phys. Rev. D, 72 (2005) 064019.

[11] Martinez C., Troncoso R. and Zanelli J., Phys. Rev. $D, \mathbf{7 0}$ (2004) 084035.

[12] Winstanley E., Class. Quantum. Grav., 22 (2005) 2233.

[13] Martinez C., Troncoso R. and Staforelli J. P., Phys. Rev. D, 74 (2006) 044028.

[14] Martinez C. and Troncoso R., Phys. Rev. D, 74 (2006) 064007.

[15] Nadalini M., Vanzo L. and Zerbini S., Phys. Rev. D, 77 (2008) 024047.

[16] Kolyvaris T., Koutsoumbas G., Papantonopoulos E. and Siopsis G., Gen. Relativ. Gravit., 43 (2011) 163.

[17] Gonzlez P. A., Papantonopoulos E., SaAvedra J. and VSQUEZ Y., JHEP, 1312 (2013) 021.

[18] Lu H., Pope C. N. and Wen Q., JHEP, 1503 (2015) 165.

[19] Cardenas M., Fuentealba O. and Martnez C., Phys. Rev. D, 90 (2014) 124072.

[20] Myung Y. S., Phys. Lett. B, 663 (2008) 111.

[21] Gegenberg J., Martinez C. and Troncoso R., Phys. Rev. D, 67 (2003) 084007.

[22] Martinez C. and Montecinos A., Phys. Rev. D, 82 (2010) 127501.

[23] Xu W., Zhao L. and Zou D. C., arXiv:gr-qc/1406.7153, (2014) .

[24] Zou D.C., Liu Y., Wang B. and Xu W., Phys. Rev. D, 90 (2014) 104035.

[25] Gonzalez P. A.,Papantonopoulos E.,SaAvedra J. and VsqueZ Y., JHEP, 1411 (2014) 011.

[26] Rao X.P., Wang B. and Yang G. H., Phys. Lett. B, 649 (2007) 472.

[27] Koutsoumbas G., Papantonopoulos E. and Siopsis G., JHEP, 0805 (2008) 107.

[28] He X., Wang B., Cai R.G. and Lin C. Y., Phys. Lett. $B, 688$ (2010) 230.

[29] Liu Y. and Wang B., Phys. Rev. D, 85 (2012) 046011

[30] Koutsoumbas G., Musiri S.,Papantonopoulos E. and SiOPSIS G., JHEP, 0610 (2006) 006.

[31] Shen J., Wang B., Lin C. Y., Cai R. G. and Su R. K., JHEP, 0707 (2007) 037.

[32] Liu Y., Zou D. C. and B. WANG, JHEP, 1409 (2014) 179.

[33] Brown J. D. And York J. W. JR., Phys. Rev. D, 47
(1993) 1407

[34] Brown J. D., Creighton J. and Mann R. B., Phys. Rev. D, 50 (1994) 6394

[35] Creighton J. D. E. and Mann R. B., Phys. Rev. D, 52 (1995) 4569

[36] Chan K. C. K. and Mann R. B., Phys. Lett. B, 371 (1996) 199

[37] Aliev A. N., Phys. Rev. D, 75 (2007) 084041

[38] Yue R., Zou D., Yu T., Li P. And Yang Z., Gen. Relativ. Gravit., 43 (2011) 2103.

[39] Cardoso V., Lemos J. P. S. and Yoshida S., Phys. Rev. D, 70 (2004) 124032.

[40] Birmingham D., Phys. Rev. D, 64 (2001) 064024.

[41] Myung Y. S. and Moon T., Phys. Rev. D, 89 (2014) 104009.

[42] Postma M. and Volponi M., Phys. Rev. D, 90 (2014) 103516.

[43] Bahamonde S., Odintsov S. D., Oikonomou V. K. and WRight M., arXiv:gr-qc/1603.05113, (2016) .

[44] Faraoni V. and Nadeau S., Phys. Rev. D, 75 (2007) 023501.

[45] Banerjee N. and Majumder B., Phys. Lett. B, 754 (2016) 129. 